\title{
Low power BPSK modulator for the application of capsule endoscope
}

\begin{abstract}
This paper presents the Binary Phase Shift Keying (BPSK) modulator for high data rate medical imaging for capsule endoscope. The BPSK modulator consists of a mixer and a ring oscillator. The ring oscillator provides carrier frequency of $433 \mathrm{MHz}$ and mix with the mixer to produce BPSK modulated signal. The modulator is designed using Silterra 0.13عm CMOS process. For supply voltage of $1.2 \mathrm{~V}$, data rate of $3.5 \mathrm{Mbps}$ the mixer has power consumption of $1.2 \mathrm{~mW}$ and at output power of $-10.7 \mathrm{dBm}$.
\end{abstract}

Keyword: BPSK; Capsule endoscope; Low power; Mixer; Modulator 